\title{
Kikuchi-Fujimoto Disease Mimicking Tuberculosis
}

\author{
Madiha Mahfoudhi*, Imen Gorsane, Sami Turki, Taieb Ben Abdallah \\ Department of Internal Medicine A, Charles Nicolle Hospital, Tunis, Tunisia \\ Email: ${ }^{*}$ madiha mahfoudhi@yahoo.fr
}

Received 10 April 2015; accepted 10 October 2015; published 13 October 2015

Copyright (C) 2015 by authors and Scientific Research Publishing Inc.

This work is licensed under the Creative Commons Attribution International License (CC BY). http://creativecommons.org/licenses/by/4.0/

(c) (i) Open Access

\begin{abstract}
A 32-year-old woman presented with a fever and cervical lymphadenopathies. The research for Mycobacterium tuberculosis was negative. Tuberculin skin test was positive. Laboratory investigations showed an elevated erythrocyte sedimentation rate and a normal white blood cell count. Bacteriological and viral investigations were negative. Quantiferon-Tb Gold test was indeterminate. Thraco-abdominal and pelvic computed tomography, FDG-PET and gallium scintigraphy revealed no abnormalities. Histological study of cervical lymph node biopsy found signs in favor of histiocytic necrotizing lymphadenitis. There were no epithelioid cell granulomas. A test tuberculosis treatment was refused by the patient. The evolution was marked by spontaneous clinical and biological resolution in fifty days. The negative Lowenstein-Jensen culture eliminated formally the tuberculosis. KFD was thus the most likely diagnosis.
\end{abstract}

\section{Keywords}

Kikuchi-Fujimoto Disease, Mycobacterium tuberculosis, Lymphadenitis, Histology

\section{Introduction}

Kikuchi-Fujimoto disease (KFD) occurring often in young women, is a histiocytic necrotizing lymphadenitis corresponding to a benign entity. It manifests frequently as lymphadenopathy and fever. Systemic manifestations may complicate this disease [1]-[3].

Exhaustive anamnesis and physical examination of an alerted clinician avoid confusion with other differential diagnosis as tuberculosis, lymphoma and sarcoidosis. Nevertheless, diagnosis confirmation is based on bacterial examination. Quantiferon-Tb Gold test may be helpful for the aetiological research in case of unexplained lymphadenopathies [4]. Histological investigation can give similar results in several pathologies and may be no con-

${ }^{*}$ Corresponding author. 
tributive [4].

KFD should be considered in the feature of lymphadenopathies associated to fever and weight loss to avoid the prescription of inappropriate treatment.

An autoimmune process, such as systemic lupus erythematosus, may occur, and thus a serious follow-up is compulsory.

The aim of this study is to emphasize on the diagnosis difficulty in case of peripheral lymphadenopathies, and to insist that KFD should be considered in these figure cases.

\section{Case Report}

A 32-year-old woman was admitted for the exploration of a prolonged fever evolving from one month. She had no personal or family history of tuberculosis. She received the BCG vaccine.

Physical examination showed lymphadenopathies, in the posterior cervical region, which diameters were between 10 and $20 \mathrm{~mm}$. Her weight was stable. He had neither splenomegaly nor hepatomegaly. The cardiac and respiratory auscultations were normal.

The research for Mycobacterium tuberculosis in her sputum and aspirated gastric liquid was negative. Tuberculin skin test was positive.

Laboratory investigations showed an elevated erythrocyte sedimentation rate $(60 \mathrm{~mm} / \mathrm{hr})$, a normal white blood cell count $(7000 / \mu \mathrm{L})$ and discrete elevated $\mathrm{C}$ reactive protein (CRP).

Bacteriological and viral researchs were negative (human immunodeficiency virus, human adult T-cell leukemia virus, hepatitis B, hepatitis C virus, Epstein-Barr virus nuclear antigen, Toxoplasma, Herpes simplex virus).

Immunological explorations were negative.

Quantiferon-Tb Gold test was undeterminate.

Thraco-abdominal and pelvic Computed tomography, FDG-PET and gallium scintigraphy revealed no abnormalities or signs suggesting a sarcoidosis, an infection or a lymphoma.

Histological study of cervical lymph node biopsy found several necrotic lesions, prominent nuclear debris and histiocytes proliferation. There were no epithelioid cell granulomas. Caseous necrosis was absent.

These results were in favor of histiocytic necrotizing lymphadenitis.

Research for microorganisms on Grocott, PAS, Ziehl-Neelsen, Gram or Warthin stains was negative eliminating many infectious diseases like tuberculosis, Whipple disease, toxoplasmosis.

A test tuberculosis treatment was proposed but refused by the patient. The evolution was marked by spontaneous disappearance of fever and lymphadenopathies and complete clinical and biological resolution in fifty days. This evolution wasn't in favor of the diagnosis of tuberculosis. The negative Lowenstein-Jensen culture eliminated formally the tuberculosis.

KFD was thus the most likely diagnosis.

\section{Discussion}

KFD corresponding to subacute necrotizing lymphadenitis manifests commonly as cervical lymphadenopathies, isolated or associated to a fever, in a young patient. It's characterized by feminine predominance. It was first described in 1972 by both Kikuchi [1], and Fujimoto and colleagues [2]. It is most frequent in Asia [1] [2].

The pathogenis of KFD is still unexplained. Several viral factors are involved as human herpes virus 6 and 8 , hepatitis B, Epstein-Barr virus and Parvovirus B19 [3]-[5].

A link between KFD and systemic lupus erythematosus was reported in many publications [3]-[5]. In our patients, viral and immunological examinations were negative.

It can be confused with lymphoma, adenocarcinoma metastasis and tuberculosis [3]. Our case was revealed by febrile cervical lymphadenopathies.

There's no pathognomonic biological test for this disease [4] [5].

Our patient presented no specific inflammatory syndrome that can be encountered in many infections and neoplastic pathologies. Tuberculosis was discussed since there were present an array of arguments (endemic country for tuberculosis, positive tuberculin test, undeterminate Quantiferon-Tb Gold test, febrile lymphadenitis).

Indeterminate result of Quantiferon-Tb Gold test may be due to decreased number and activity of $\mathrm{T}$ lympho- 
cytes or the failure of IFN- $\gamma$ synthesis in T lymphocytes. A study of T lymphocyte wasn't done in our patient.

The presence of epithelioid cell granulomas with caseous necrosis on histological results of lymph node biopsy is in favour of the diagnosis. An histiocytic lymphadenitis is commonly present in KFD but can be also encountered in tuberculosis [6].

Only the absence of Mycobacterium tuberculosis by using Ziehl-Neelsen coloration or Lowenstein-Jensen culture permits to eliminate tuberculous origin of lymphadenitis; this is avoid abusive prescription of antituberculous treatment characterized by many side effects [6]-[8]. In our case, anti-tuberculous therapy was indicated but refused by the patient.

The spontaneous clinical and biological improvement (disappearance of lymphadenopathies and biological inflammatory syndrome) in our case was against tuberculosis diagnosis, corticosteroid therapy wasn't required in our patient.

KFD can also be associated to systemic lupus erythematosus hence the interest of immunologic investigation in case of KFD diagnosis [9].

In our observation, all immunologic tests were negative eliminating this association and the occurrence of other autoimmune disease.

A study described the clinicopathological features of 40 cases of necrotizing non-granulomatous lymphadenitis [10]. Of the 40 patients, 17 cases (42.5\%) had KFD, 8 cases (20\%) had tuberculosis lymphadenitis and 1 case (2.5\%) had systemic lupus erythematosus with associated lymphadenitis. KFD is characterized by the presence of coagulative necrosis and karyorrhexis often centered in the paracortex, an absence of neutrophils and plasma cells, proliferation of various cells composed of lymphocytes, histiocytes, immunoblasts and plasmacytoid monocytes and the absence of a granuloma [10].

The evolution of KFD is usually benign and followed by complete resolution spontaneously or within several months of corticosteroid therapy. In our case, all signs resolved spontaneously [6]-[8].

KFD is therefore a particular entity that should be considered by the physician.

\section{Conclusion}

KFD is a rare diagnosis that should be evoked to avoid its confusion with serious affections as lymphoma, tuberculosis, metastasis, having different clinical course and requiring specific therapy.

\section{Disclosure Statement}

The authors have nothing to disclose.

\section{References}

[1] Kikuchi, M. (1972) Lymphadenitis Showing Focal Reticulum Cell Hyperplasia with Nuclear Debris and Phagocytosis. Nippon Ketsueki Gakkai Zasshi, 35, 379-380.

[2] Fujimoto, Y., Kozima, Y. and Yamaguchi, K. (1972) Cervical Subacute Necrotizing Lymphadenitis: A New Clinicopathological Entity. Naika, 30, 920-927.

[3] Xavier, R.G., Silva, D.R., Keiserman, M.W. and Lopes, M.F. (2008) Kikuchi-Fujimoto Disease. Jornal Brasileiro de Pneumologia, 34, 1074-1078. http://dx.doi.org/10.1590/S1806-37132008001200014

[4] Nakamura, I., Imamura, A., Yanagisawa, N., Suganuma, A. and Ajisawa, A. (2009) Medical Study of 69 Cases Diagnosed as Kikuchi’s Disease. Kansenshogaku Zasshi, 83, 363-368.

[5] Bosch, X., Guilabert, A., Miquel, R. and Campo, E. (2004) Enigmatic Kikuchi-Fujimoto Disease: A Comprehensive Review. American Journal of Clinical Pathology, 122, 141-152. http://dx.doi.org/10.1309/YF081L4TKYWVYVPQ

[6] Kurahara, Y., Tachibana, K., Tezuka, K., Shimizu, S., Kitaichi, M., Hayashi, S., et al. (2012) Kikuchi-Fujimoto Disease Mimicking Tuberculous Lymphadenitis. Internal Medicine, 51, 1927-1930. http://dx.doi.org/10.2169/internalmedicine.51.7574

[7] Nayak, H.K., Mohanty, P.K., Mallick, S. and Bagchi, A. (2013) Diagnostic Dilemma: Kikuchi’s Disease or Tuberculosis? BMJ Case Reports, 2013. http://dx.doi.org/10.1136/bcr-2012-008026

[8] Kamath, M.P., Bhojwani, K., Naik, R., Kumar, R. and Chakravarthy, Y. (2006) Tuberculosis Mimicking Kikuchi’s Disease. Ear, Nose \& Throat Journal, 85, 126-128. 
[9] el-Ramahi, K.M., Karrar, A. and Ah, M.A. (1994) Kikuchi Disease and Its Association with Systemic Lupus Erythematosus. Lupus, 3, 409-411. http://dx.doi.org/10.1177/096120339400300508

[10] Sanpavat, A., Wannakrairot, P. and Assanasen, T. (2006) Necrotizing Non-Granulomatous Lymphadenitis: A Clinicopathologic Study of 40 Thai Patients. Southeast Asian Journal of Tropical Medicine and Public Health, 37, 563-570. 